\title{
Publisher Correction: Biotech news from around the world
}

Correction to: Nature Biotechnology https://doi.org/10.1038/s41587-021-01082-4, published online 7 October 2021.

In the version of this article initially published, there was a typo in the map illustration. In the center-right box for Japan, the text now reading "Kindai University" initially appeared as Kinkai University. The error has been corrected in the online version of the article.

Published online: 26 October 2021

https://doi.org/10.1038/s41587-021-01134-9

๑) Springer Nature America, Inc. 2021 